MODELING, IDENTIFICATION AND CONTROL, 1981, VOL. 2, NO. 2, 71-82

doi: $10.4173 /$ mic.1981.2.2

\title{
Pressure and flow in arterial aneurysms simulated in mathematical models
}

\author{
SVEN ØIVIND WILLE†
}

Keywords: finite element method, blood flow, aneurysms, arteries.

In this work pressure and flow patterns inside two kinds of axis symmetric aneurysms are presented under steady flow conditions. The results are obtained by solving the axi-symmetric formulation of the non-linear Navier-Stokes equations.

The technique used to solve the equation is the finite element method. The results are given at various Reynolds numbers up to 500, and displayed as pressure isobars, streamlines and velocity vectors. For the higher Reynolds numbers vortex formation in the aneurysms is demonstrated. The pressure gradient is found to be small inside the aneurysms compared to those at the inlet and outlet tubes.

\section{Introduction}

The factors which lead to the development of aneurysms are poorly understood, but atherosclerotic disease is the most common cause. It is an interesting finding that aneurysm, and occlusive arterial disease rarely occur in the same individual (Spittell 1971). Aneurysms are subdivided, according to their shape, into saccular and fusiform, although this distinçtion is not always clear. The saccular type is a pouch-like expansion of a localized area of the wall, often found at the apex of bifurcations. The fusiform aneurysm is a radial dilatation of an arterial segment with a size up to several times the original vessel diameter. The gradual enlargement of an aneurysm is usually accompanied by a development of a laminated thrombus within the aneurysm, although a central channel of flow is maintained. The presence of thrombus may lead to distal embolization through release of thrombotic fragments.

There have been relatively few investigations of pressure and flow patterns inside fusiform aneurysms. Scherer (1973) investigated the flow patterns in glass models of aneurysms for an asymmetrical aneurysm and an axis symmetrical spherical aneurysm under steady flow conditions. By injecting dye into the fluid the streamlines were visualized and with pressure manometers proximal and distal to the aneurysm, the pressure drop was obtained. The results showed vortex formation in the dilated part of the aneurysm with a fluid core passing through in the centre. The cross-sectional areas of the fluid core decreased as the flow rate was increased and there was a slight pressure drop of the order of $1 \mathrm{~cm} \mathrm{H} \mathrm{H}_{2} \mathrm{O}$ across the aneurysm. At the onset of turbulence, the pressure gradient increased substantially.

The purpose of the present work is to obtain a detailed description of pressure and flow patterns inside two kinds of fusiform aneurysms by a numerical technique, the finite element method. The results are obtained by solving the axi-symmetrical formulation of the Navier-Stokes equations and they are presented at various Reynolds numbers for steady flow conditions.

Received 21 October 1980.

$\dagger$ Institute of Informatics, University of Oslo, Norway. 
The advantage of such a model is that it is possible to extend the analysis to the more realistic condition of a time dependent flow system.

\section{Mathematical methods}

The well-known Navier-Stokes equations and the continuity equation governing axi-symmetric steady flow are as follows:

$$
\begin{gathered}
\frac{1}{\rho} \frac{\partial p}{\partial x}-\mu\left(\frac{1}{r} \frac{\partial u}{\partial r}+\frac{\partial^{2} u}{\partial x^{2}}+\frac{\partial^{2} u}{\partial r^{2}}\right)+u \frac{\partial u}{\partial x}+v \frac{\partial u}{\partial r}=0 \\
\frac{1}{\rho} \frac{\partial p}{\partial r}-\mu\left(\frac{1}{r} \frac{\partial v}{\partial r}-\frac{v}{r^{2}}+\frac{\partial^{2} v}{\partial x^{2}}+\frac{\partial^{2} v}{\partial r^{2}}\right)+u \frac{\partial v}{\partial x}+v \frac{\partial v}{\partial r}=0 \\
\frac{v}{r}+\frac{\partial u}{\partial x}+\frac{\partial v}{\partial r}=0
\end{gathered}
$$

where $u=$ velocity in $x$ (longitudinal) direction, $v=$ velocity in $r$ (radial) direction, $p=$ pressure, $\rho=$ density of the fluid and $\mu=$ kinematic viscosity.

In applying these equations for blood flow, certain assumptions are made. The blood is treated as a homogeneous, Newtonian fluid and the blow flow is assumed to be laminar and isothermal. The terms appearing in the Navier-Stokes equations are the pressure gradient and viscous terms which are linear and the non-linear convective acceleration. The non-linearities in these equations increase as the Reynolds number increases and therefore the convective acceleration is the limiting factor in obtaining a convergent solution.

The technique used to solve the simultaneous equations is the finite element method. In applying this method, the fluid domain is divided into isoparametric triangles on which the pressure and velocities are approximated by a series expansion. The pressure is interpolated by a linear polynomial and the velocities by quadratic polynomials. The finite element formulation is obtained by applying the Galerkin weighted residual process (Zienkiewicz 1971) and the contribution to the equation system from each triangle is assembled into a global element matrix. The non-linear equations formed by this procedure are solved by the iterative Newton-Raphson technique. At each iteration, a Gauss elimination equation solver is used to obtain a correction to the solution from the previous step. When the relative correction becomes acceptably small, the iteration procedure is stopped. The streamlines are calculated from the velocity field by applying the finite element method once more. The definition of the stream function for axi-symmetric flow is given by

$$
\begin{aligned}
& u=-\frac{1}{r} \frac{\partial \psi}{\partial r} \\
& v=\frac{1}{r} \frac{\partial \psi}{\partial x}
\end{aligned}
$$

Differentiating and adding the expressions together gives

$$
\frac{\partial^{2} \psi}{\partial x^{2}}+\frac{\partial^{2} \psi}{\partial r^{2}}=r \frac{\partial v}{\partial x}-r \frac{\partial u}{\partial r}-u
$$


The right-hand side of this equation is known from previous calculations. The streamline equation is linear and easily solved. Special software is designed to provide the plots of pressure, streamlines and velocity vectors. A description of the finite element equations and solution procedure is given by Wille (1980).
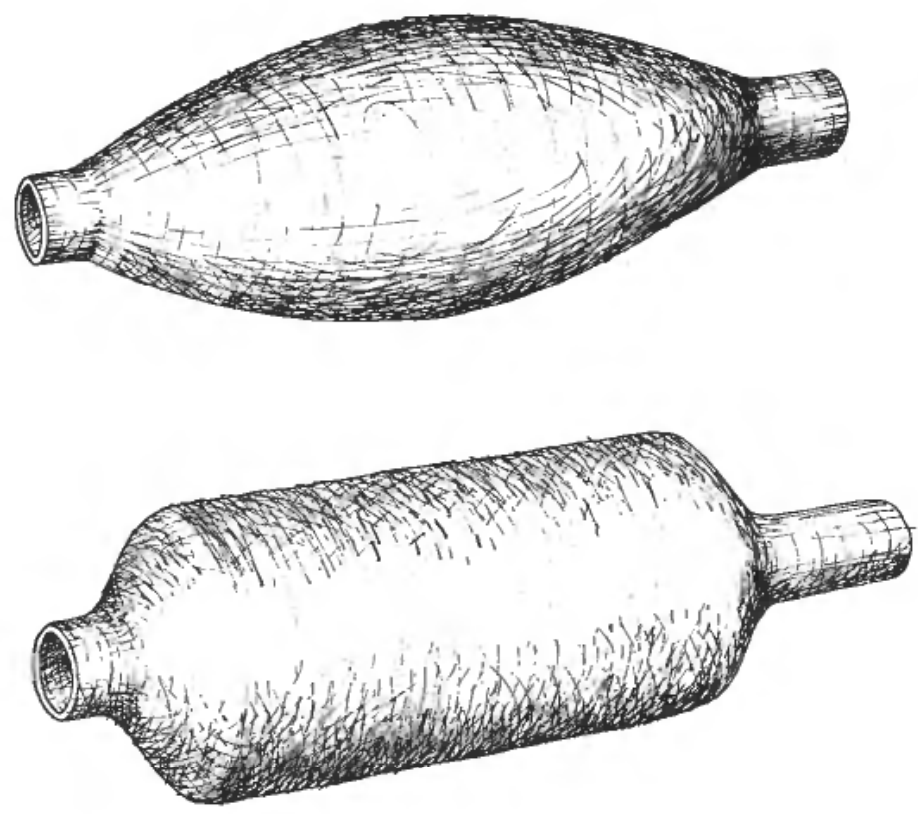

Figure 1. The two kinds of aneurysms simulated, the upper with smooth corners, and the lower with sharp corners.

\section{ANEURYSM MODELS}

$\mathrm{u}=0 \quad \mathrm{v}=0$

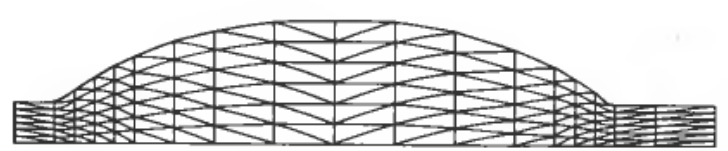

$u=1-y^{2} / R^{2}$
$v=0$

$\frac{\partial u}{\partial n}=0 \quad \frac{\partial v}{\partial n}=0$

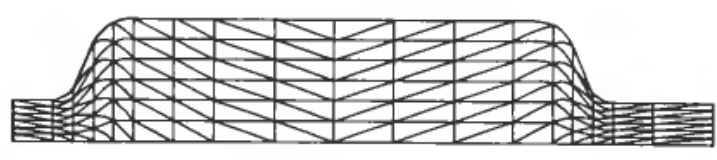

$v=0 \quad \frac{\partial u}{\partial n}=0$

Figure 2. The finite element models of the aneurysms with boundary conditions. The boundary conditions, $u=0, v=0$ correspond to the wall, the boundary conditions $v=0, \partial u / \partial n=0$ correspond to the axis of symmetry, the boundary conditions $\partial u / \partial n=0$, $\partial v / \partial n=0$ correspond to the outlet and $u=1-y^{2} / R^{2}, v=0$ correspond to the inlet.

M.I.C. 


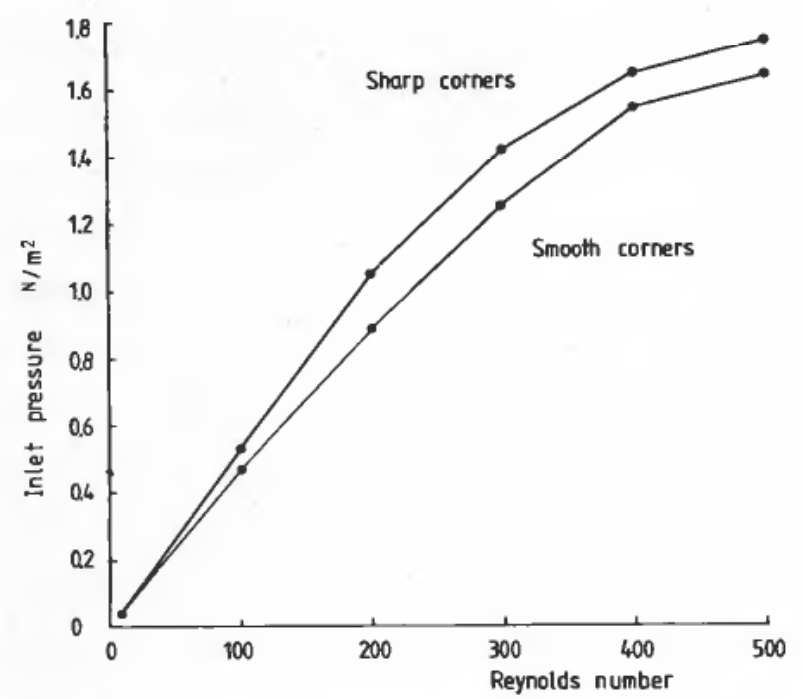

Figure 3. The inlet pressure of the two aneurysm models as a function of Reynolds number. The inlet pressure is the same as the pressure gradient as the pressure at the outlet is specified to be zero. The pressure gradient is increasing with Reynolds number and it is higher for the aneurysm with sharp corners.
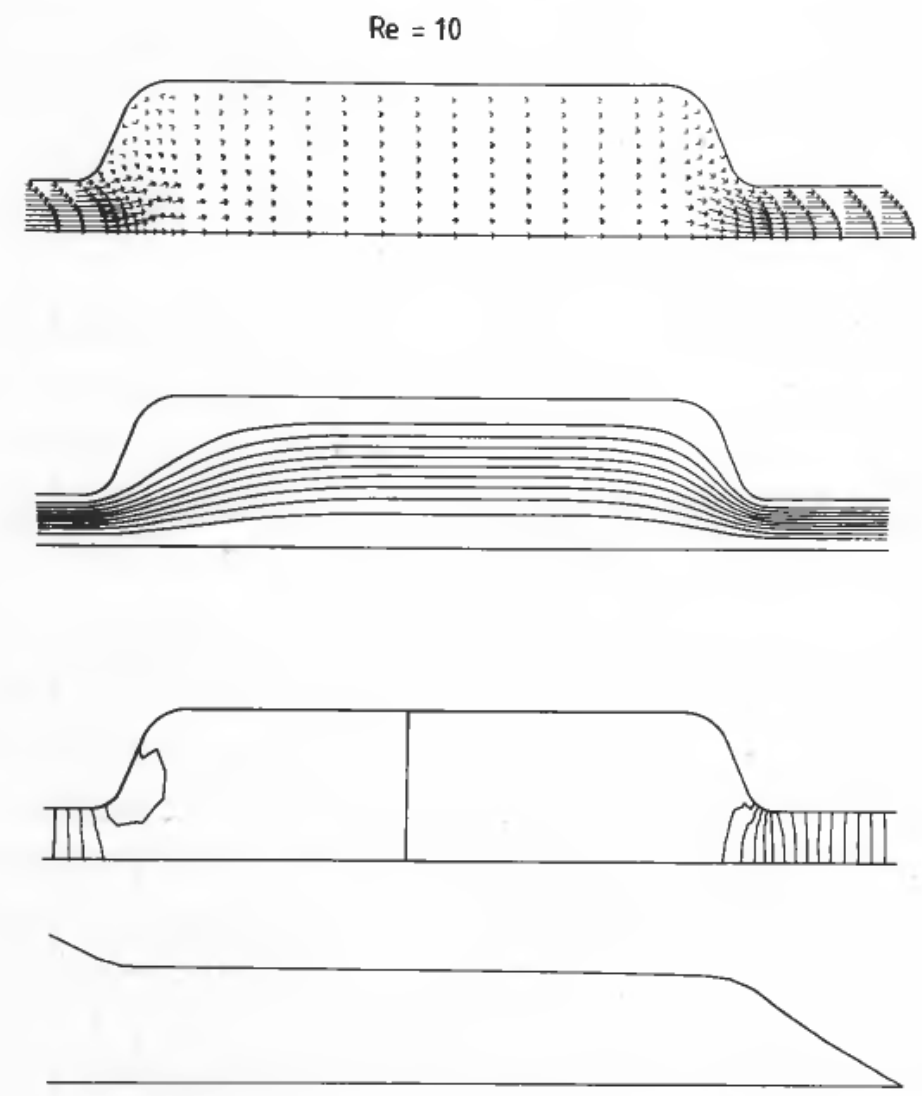

Figure 4. The velocity vectors, streamlines, isobars and pressure variation along the central axis for Reynolds number 10 . 


\section{Models and boundary conditions}

Two models of the fusiform type of aneurysm are considered, one with smooth corners and the other with sharp corners. The radius of the maximum expansion of each aneurysm is three times that of the radius at the inlet and outlet; the cross-sectional area of the expansion is therefore nine times that of the inlet and outlet.

Due to the axial symmetry of the aneurysms, it is sufficient to simulate only one half of the model through the plane of symmetry. The finite element models of the aneurysms with their triangular meshes are shown in Fig. 2. As the high pressure and flow gradients are expected at the inlet and outlet, the triangular mesh is made finer in these zones. The boundary conditions at the inlet are a parabolic velocity profile $\left(u=1-y^{2} / R^{2}\right)$ in the longitudinal direction and zero velocity $(v=0)$ in the radial direction. At the rigid wall, the velocities are zero $(u=0, v=0)$ to fulfil the no slip condition. Along the axis of symmetry, the radial component $(v=0)$ as well as the normal derivative of the tangential velocity $(\partial u / \partial n=0)$ are zero. The outlet boundary condition is zero normal derivative of both components of the velocity $(\partial u / \partial n=0, \partial v / \partial n=0)$.

$\operatorname{Re}=100$
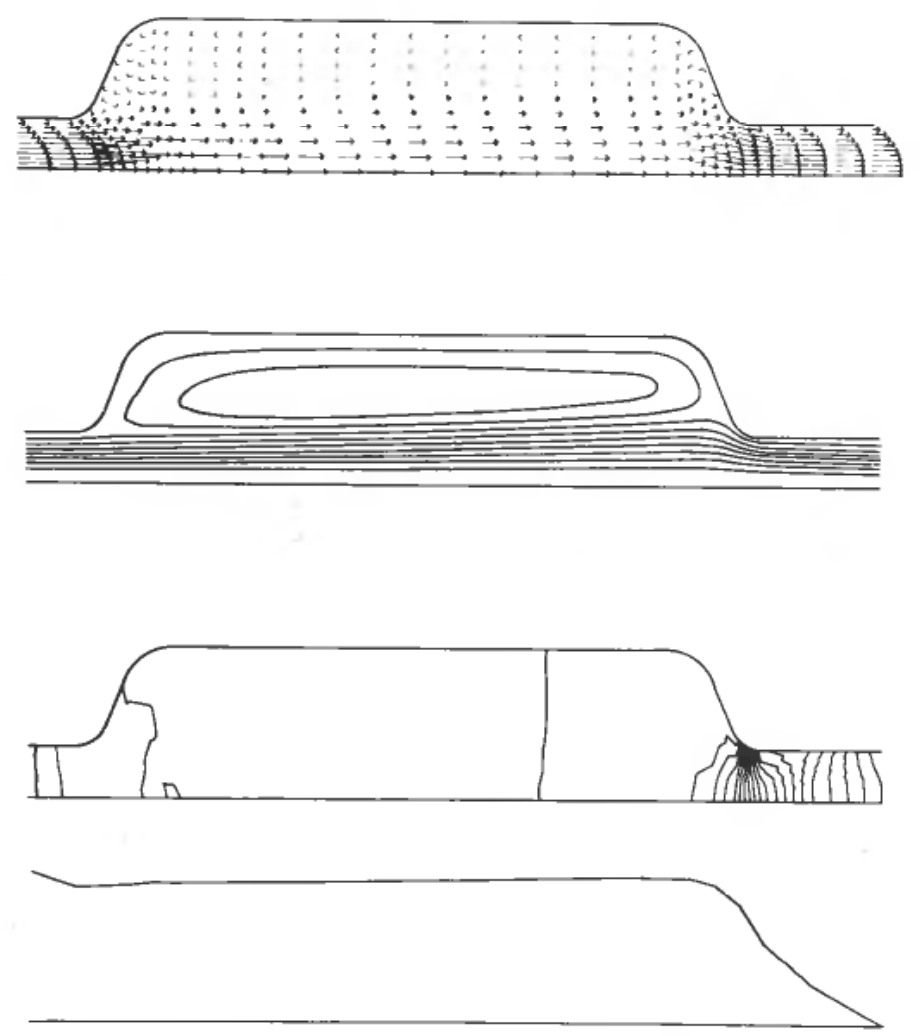

Figure 5. The velocity vectors, streamlines, isobars and pressure variation along the central axis for Reynolds number 100. 
The Reynolds number is defined by

$$
\operatorname{Re}=\frac{\bar{u} \cdot d}{\mu}
$$

where $\bar{u}=$ mean inlet velocity, $d=$ inlet diameter and $\mu=$ kinematic viscosity. In these investigations, the Reynolds numbers given are those calculated at the inlet. Using the above formula, the Reynolds number at the expansion is one third of the inlet Reynolds number. Changes in the Reynolds number are made by alteration of the inlet velocity. The pressure at the outlet of the model is taken to be zero at the outlet walls. The inlet radius of both aneurysm models is $R=1 \times 10^{-2} \mathrm{~m}$ and the kinematic viscosity $\mu=1 \times 10^{-6} \mathrm{~m}^{2} \mathrm{~s}^{-1}$.

\section{Results}

The analyses of the aneurysm models has been carried out for several Reynolds numbers. The inlet pressure, which is equal to the pressure gradient since the pressure is specified to be zero at the outlet, is shown as function of the Reynolds number. The Reynolds numbers 10, 100, 200,300, 400, 500 correspond to the central inlet

$$
\operatorname{Re}=200
$$
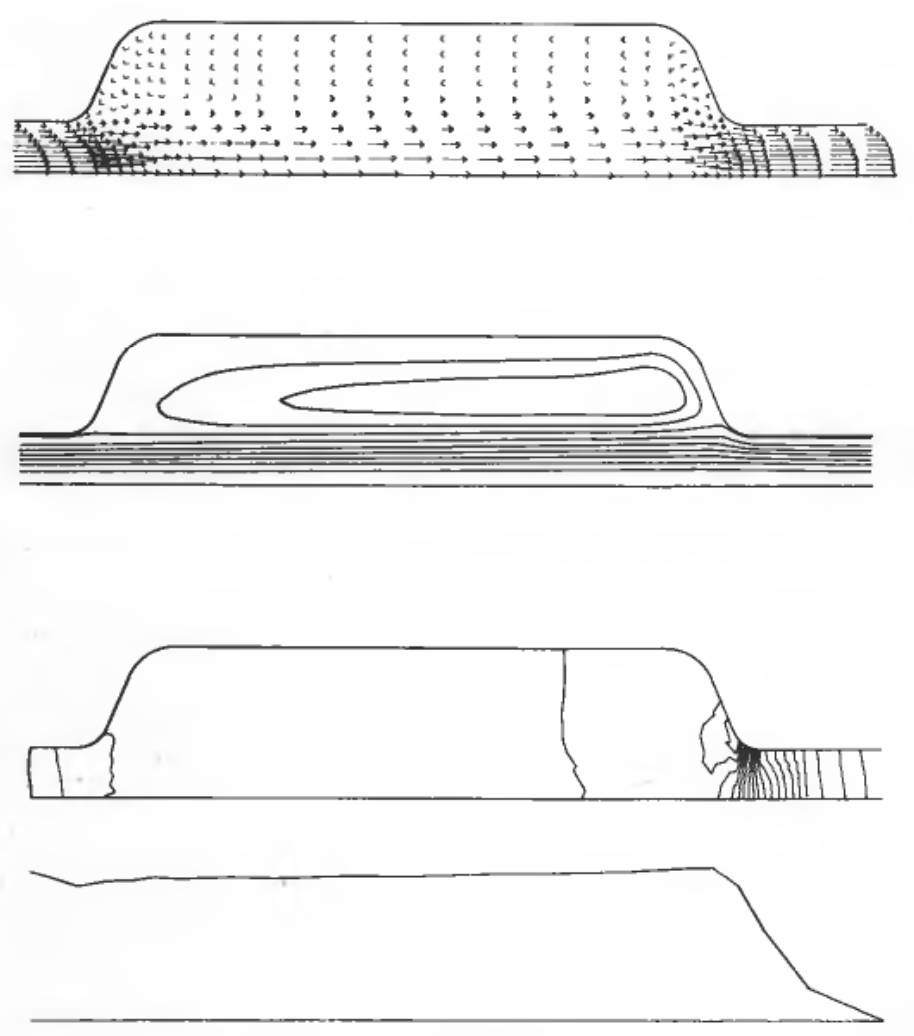

Figure 6. The velocity vectors, streamlines, isobars and pressure variation along the central axis for Reynolds number 200. 
velocities equal to $4 \times 10^{-3}, 4 \times 10^{-2}, 6 \times 10^{-2}, 8 \times 10^{-2}, 1 \times 10^{-1}, 1 \cdot 2 \times 10^{-1}$ respectively. As seen from the figure, the pressure drop across the aneurysm with sharp corners is greater than the pressure drop across the aneurysm with smooth corners for Reynolds numbers above 10.

In Figs. 4-11 the pressure and flow patterns for the two aneurysm models are shown. In these figures the diagrams from top to bottom show the velocity vectors, the streamlines, the isobars and the pressure variation along the central axis of the aneurysm. The velocity vectors indicate the direction of the flow and the magnitude of the velocity. The scaling factor for the velocity vectors at each Reynolds number is given above. The streamlines are equally spaced and indicate the path of the fluid particles. The isobars are lines drawn through points in the fluid with the same pressure. The pressure differences between the isobars are constant. The pressure value on each isobar is easily found from the pressure curve along the central axis of the aneurysm below. The values of the inlet pressures are found in Fig. 3.

The simulation for Reynolds number 10 of the aneurysm with sharp corners is found in Fig. 4. It may be seen that there is a linear pressure fall at the inlet and outlet. Just after the start of the expansion there is a local pressure minimum at the wall. In the aneurysm itself there is almost no change in pressure. The streamline plot as well as the velocity plot show that the velocities are parallel to the boundary wall.

$\operatorname{Re}=300$
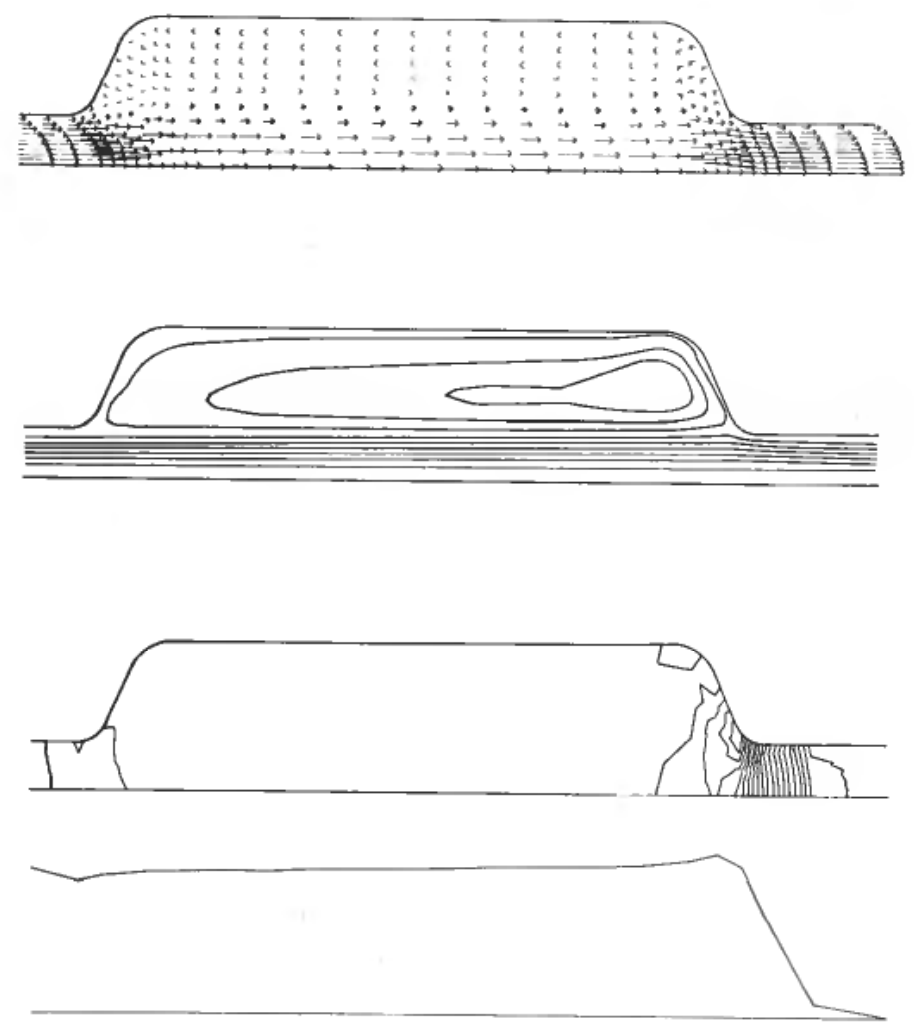

Figure 7. The velocity vectors, streamlines, isobars and pressure variation along the central axis for Reynolds number 300. 
At the Reynolds number of 100, Fig. 5, the largest pressure gradients, as indicated by the closeness of the isobars, still occur at the inlet and outlet. There is a relatively small pressure gradient within the expansion. The streamlines indicate a large vortex which occupies almost the entire expansion. The centre of the vortex is found in the middle of the expansion.

As the Reynolds number is increased, the centre of the vortex moves downstream. There is also an increase in the energy within the vortex since the number of rings inside it have increased.
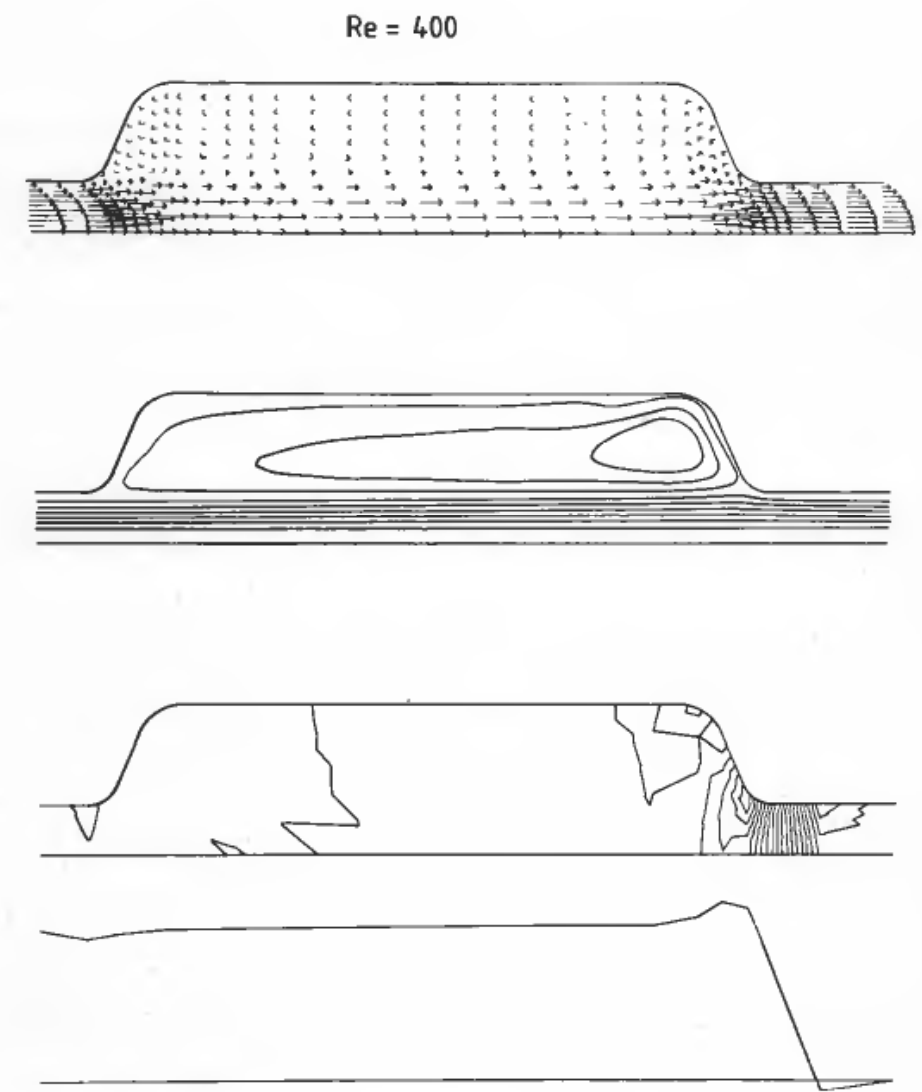

Figure 8. The velocity vectors, streamlines, isobars and pressure variation along the central axis for Reynolds number 400 .

At a Reynolds number of 500, Fig. 9, the main pressure gradient occurs on the outlet side and a local high pressure zone has developed at the downstream wall. The pressure inside the aneurysm is almost constant. The vortex has slightly increased in size, whilst the centre of the vortex has moved farther downstream. The majority of the streamlines at the entrance of the expansion continue in the central core of the expansion and pass into the outlet channel.

The results of the simulation for the model with smooth corners for Reynolds number 10 and 500 are shown in Figs. 10-11. The general features of the pressure and flow patterns are similar to those found for the model with more sharp corners. 

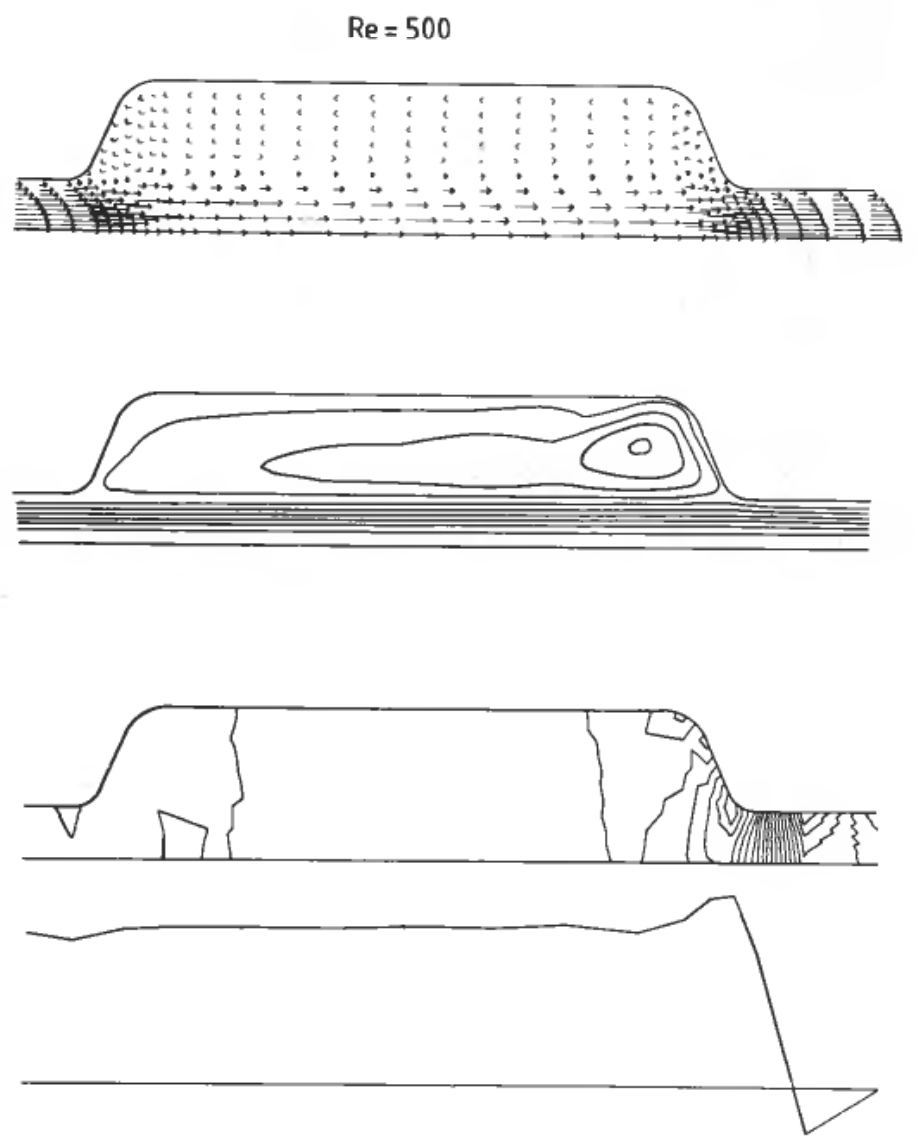

Figure 9. The velocity vectors, streamlines, isobars and pressure variation along the central axis for Reynolds number 500 .

\section{Discussion}

In the work described above, some simplifications and assumptions have been made. Firstly, the models have been assumed to be rigid. The viscoelastic properties of the normal aorta have been investigated by a number of workers, see for example, Peterson, Jensen and Parnell (1960), Learoyd and Taylor (1966) and Patel, Janici and Carew (1969). Although the distensibility has been shown to depend on a number of factors such as age and intralumenal pressure, it would seem that, in general, the radial movement produced by the pressure pulsation is only a small percentage of the radius of the vessel. Thus, to a first approximation, at least, the assumption of wall rigidity is reasonably valid. It is also assumed that blood is homogeneous; this is of course not the case, but it has been shown (McDonald 1974) that in vessels which are large in comparison to the size of the red blood corpuscles it may be considered homogeneous and Newtonian, that is, with a viscosity independent of shear rate.

The assumption of steady flow conditions is an extreme oversimplification in view of the pulsatile nature of arterial flow. However, the presentation of the mathematical analysis of pressure and flow patterns under steady flow conditions may be regarded as an initial step. 

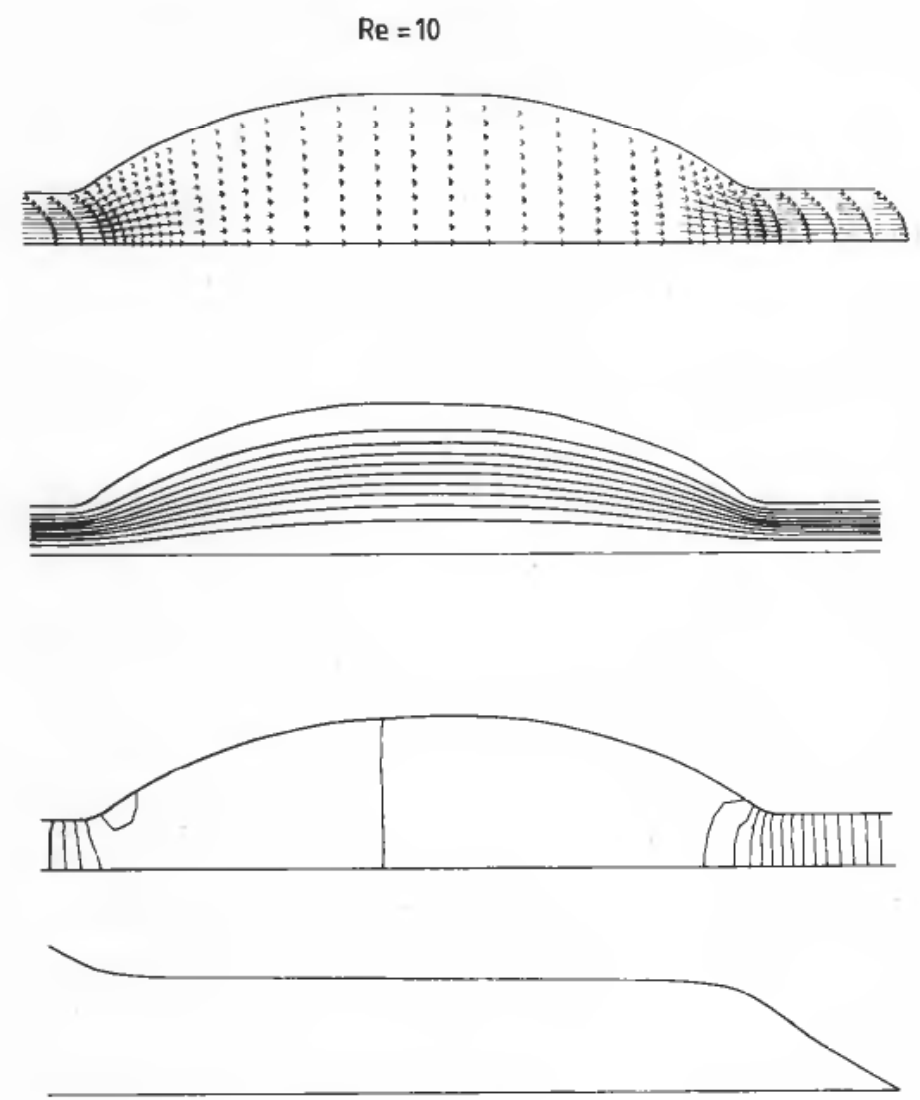

Figure 10. The velocity vectors, streamlines, isobars and pressure variation along the central axis for Reynolds number 10.

The development of a vortex with the increase in Reynolds number is well demonstrated by the analysis. At a Reynolds number of 500 which is typical of the Reynolds number of the steady flow component of aortic flow, the vortex centre is displaced toward the distal end of the expansion. The results obtained by Scherer (1973) in a glass model with a spherical aneurysm showed similar vortex patterns.

The presence of a vortex with a slow circulation of fluid and only a central core of forward flow will contribute to the formation of the thrombus which is known to develop in large aneurysms.

The pressure gradient within the aneurysm is small as found by Scherer (1973); however, the analysis indicates the pressure contours in more detail than could be achieved experimentally, and for example demonstrates the presence of a local high pressure region close at the distal wall of the aneurysm at the highest Reynolds number examined. This high pressure zone will tend to expand an already existing aneurysm farther downstream.

The effect of changing the geometrical shape of the aneurysm is minimal although as would be expected the vortex centre is displaced farther towards the distal end of the aneurysm at the higher Reynolds numbers. 

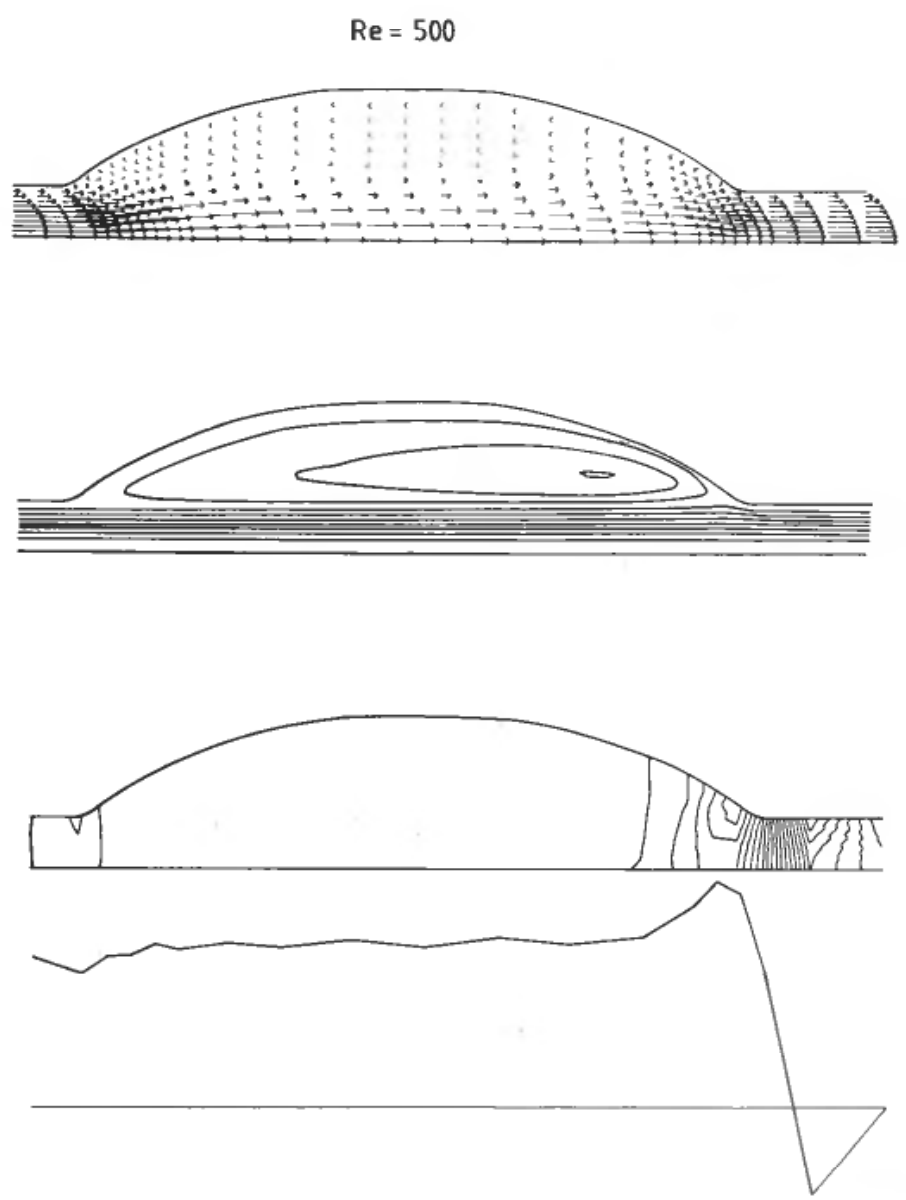

Figure 11. The velocity vectors, streamlines, isobars and pressure variation along the central axis for Reynolds number 500 .

It is hoped that this paper has shown that the finite element analysis to determine the pressure and flow fields can reveal details which would not be obtainable by experimental techniques.

\section{ACKNOWLEDGMENT}

The author is grateful to Lars Walløe for discussions and encouragement.

\section{REFERENCES}

LeAROYD, B. M., and TAYLOR, M. G. (1966). Alterations with age on the viscoelastic properties of human arterial walls. Circ. Res., 18, 278-292.

MCDonald, D. A., (1974). Blood Flow in Arteries (Southampton: The Camelot Press Ltd.).

Patel, D. J., JANICI, J. S., and Carew, T. E. (1969). Static anisotropic elastic properties of of the aorta in living dogs. Circ. Res., 25, 765-779.

Peterson, L. H., Jensen, R. E., and Parnell, J. (1960). Mechanical properties of arteries in vivo. Circ. Res., 8, 622-639.

SCHERER, P. W., (1973). Flow in axisymmetrical glass model aneurysms. J. Biomechanics, $6,695-700$. 
Spittell, J. A. Jr. (1971). Aortic and Peripheral Arterial Aneurysms. Peripheral Vascular Disease, Vol. 3, No. 1 (edited by R. W. Gifford, Jr.) (Philadelphia: F. A. Davis Company).

WiLLE, S. Ø. (1980). A finite element solution of the Navier-Stokes equations for twodimensional and axi-symmetric flow. Modeling, Identification and Control, 2, 105117.

ZiENkIEwicz, O. C. (1971). The Finite Element Method in Engineering Science (London: McGraw-Hill). 